\title{
Clinical and Prognostic Implications of an Immune- Related Risk Model Based on TP53 Status in Lung Adenocarcinoma
}

\section{Xuming Song}

Jiangsu Institute of Cancer Research: Jiangsu Cancer Hospital

\section{Qiang Chen}

Xuzhou Central Hospital

\section{Jifan Wang}

Jiangsu Institute of Cancer Research: Jiangsu Cancer Hospital

\section{Qixing Mao}

Jiangsu Institute of Cancer Research: Jiangsu Cancer Hospital

\section{Wenjie Xia}

Jiangsu Institute of Cancer Research: Jiangsu Cancer Hospital

\section{Lin Xu}

Jiangsu Institute of Cancer Research: Jiangsu Cancer Hospital

\section{Feng Jiang}

Jiangsu Institute of Cancer Research: Jiangsu Cancer Hospital

Gaochao Dong ( $\square$ ilsyvm@njmu.edu.cn )

Jiangsu Institute of Cancer Research: Jiangsu Cancer Hospital https://orcid.org/0000-0002-7026-6280

\section{Primary research}

Keywords: Lung adenocarcinoma, TP53 mutation, Immune prognostic model, Immune profile

Posted Date: November 17th, 2020

DOI: https://doi.org/10.21203/rs.3.rs-105337/v1

License: (c) (1) This work is licensed under a Creative Commons Attribution 4.0 International License.

Read Full License

Version of Record: A version of this preprint was published at Journal of Cellular and Molecular Medicine on December 8th, 2021. See the published version at https://doi.org/10.1111/jcmm.17097. 


\section{Abstract}

Background: TP53 mutation is the most widespread mutation in lung adenocarcinoma (LUAD), meanwhile p53 (encoded by TP53) has recently been implicated in immune responses. However, it is still unknown whether TP53 mutation may remodel tumor microenvironment to influence tumor progression and prognosis in LUAD.

Methods: we developed a six-gene immune-related model (IRM) to predict the survival of patients with LUAD in TCGA cohort based on TP53 status using LASSO Cox analysis, which was also confirmed the predictive ability in two independent cohorts.

Results: The mutation of TP53 led to a decrease in the immune response in LUAD. Further analysis revealed that patients in the high-index group had observably lower relative infiltration of memory B cells and regulatory $T$ cells, together with significantly higher relative infiltration proportions neutrophils and resting memory $\mathrm{CD} 4^{+} \mathrm{T}$ cells. Additionally, the IRM index positively correlated with expression of critical immune checkpoint genes including PDCD1 (encoding PD-1) and CD274 (encoding PD-L1), which was validated in Nanjing cohort. Furthermore, the IRM index as an independent prognostic factor was used to establish a nomogram for clinical application.

Conclusion: This immune-related model may serve as a powerful prognostic tool to further optimize immunotherapies for LUAD.

\section{Introduction}

Non-small cell lung cancer (NSCLC) accounts for $85 \%$ of all lung cancers, and lung adenocarcinoma (LUAD) is the most frequent NSCLC subtype ${ }^{1}$. Immunotherapy has been integrated into the first- and second-line treatment strategies for NSCLC, reviving enthusiasm in explaining the prognostic and pathophysiological role of the tumor microenvironment $(\mathrm{TME})^{2-5}$. However, it is not known whether the immune signature of lung cancer could act as a biomarker to reliably estimate disease prognosis and patient survival.

TP53 is a tumor suppressor gene, and it is one of the 5 most conspicuous mutations in LUAD, though there has not yet been approval for any related molecular inhibitors by the Food and Drug Administration. TP53 encodes p53, a master regulatory transcription factor that controls multiple core programs in cells, including cell cycle arrest, apoptosis, senescence, fertility, and metabolism ${ }^{6-10}$. Recently, p53 has been implicated in immune responses ${ }^{11}$. TP53 mutation remarkably affects the expression of immune checkpoints and activated $\mathrm{T}$ cell immune response and may thus serve as a potential predictive factor for guiding immunotherapy ${ }^{11}$. However, the relationship between TP53 mutation and the regulation of immune signaling and responses is still unknown. We hypothesized that TP53 mutation may remodel the TME to influence tumor progression and prognosis in LUAD. 
Recently, computational methods based on transcriptome data were proposed to characterize the immune landscape in the sequenced tumor tissue ${ }^{12}$. In this study, we systemically screened the expression profiles of RNA sequencing data from public databases and developed a 6-gene immunerelated risk model (IRM) based on TP53 mutation to predict the survival of patients with LUAD. We further validated the 6-gene IRM index in the meta-Gene Expression Omnibus (GEO) and Nanjing cohorts. Through multiple verification methods, we determined the IRM index to be an independent prognostic biomarker that could accurately predict the 5 - and 7-year overall survival (OS) of patients with LUAD. The potential mechanism, immune infiltration, and expression of immune-related checkpoints of the IRM index were also explored. We believe that this robust prognostic IRM index will improve risk stratification, provide more exact judgment for individualized clinical management, and serve as a potential immunotherapy biomarker for patients with LUAD.

\section{Materials And Methods}

\subsection{RNA sequencing data and immune-related genes}

The nonsynonymous mutation status of 512 patients with LUAD (workflow type: Mutect2 pipeline), gene expression data (workflow type: HTSeq-Counts), and corresponding clinical metadata from The Cancer Genome Atlas (TCGA) website (https://gdc.cancer.gov/) were downloaded using the "TCGAbiolinks" R package (version 2.14.1; https://bioconductor.org/packages/release/bioc/html/TCGAbiolinks.html). Of these patients, 499 with RNA sequencing data and TP53 mutation status data were included in the following analysis. Using the "maftools" R package (version 2.2.10; https://www.bioconductor.org/packages/release/bioc/html/maftools.html), we identified the mutation status of these patients, including 239 TP53 ${ }^{\text {MUT }}$ patients and 260 TP53 ${ }^{\text {WT }}$ patients. Entrez IDs were converted to gene symbols using the Bioconductor package "org.Hs.eg.db" (version 3.10.0; https://bioconductor.org/packages/release/data/annotation/html/org.Hs.eg.db.html). Genes with low abundance expression were removed from the profile.

\subsection{Differential expression analysis}

Differential expression analysis was performed using the "DESeq2" R package (version 1.26.0; https://bioconductor.org/packages/release/bioc/html/DESeq2.html) with the standard comparison mode between the 2 experimental conditions. To filter for differentially expressed genes (DEGs) between TP53 $^{\text {mut }}$ and TP53 ${ }^{\text {wt }}$ patients, log2|fold change| $>1$ and adj. $P<0.05$ were set as the cut-point.

\subsection{Construction of the IRM index}

Immune-related differentially expressed genes (IRDEGs) were collected from the ImmPort gene list (http://www.immport.org), which is a gene set sponsored by the National Institute of Allergy and Infectious Diseases (NIAID) that includes annotated genes related to immune activity processes. After identifying IRDEGs, a least absolute shrinkage and selector operation (LASSO) algorithm was built using the "glmnet" R package (version 3.0; https://cran.r-project.org/web/packages/glmnet/index.html) to 
select candidate genes. Kaplan-Meier survival curves were created using the "survival" R package (version 3.5; https://cran.r-project.org/web/packages/survival/index.html). Finally, the "timeROC" R package (version 0.4; https://cran.r-project.org/web/packages/timeROC/index.html) was used to conduct a timedependent receiver operating characteristic (ROC) curve analysis.

\subsection{Microarray data}

The gene expression matrixes from GSE29013, GSE30219, and GSE31908 based on the GPL570 platform, including 131 patients with LUAD, were downloaded from the GEO database (https://www.ncbi.nlm.nih.gov/gds). The gene expression data for the 3 matrixes were subjected to $\log _{2}$ transformation. The scale method of the "limma" R package (version 3.42.2; https://bioconductor.org/packages/release/bioc/html/limma.html) was used to normalize the data.

\subsection{Patients in the Nanjing cohort and sample collection}

To further evaluate the clinical effectiveness of the IRM index, we enrolled a cohort including 92 patients who underwent radical surgery without neoadjuvant chemotherapy and who were diagnosed with LUAD at the Jiangsu Cancer Hospital (Nanjing, China). The patients' characteristics are presented in Table S1. The patients in the Nanjing cohort were represented by formalin-fixed, paraffin-embedded (FFPE) specimens collected from radical surgery from 2012 to 2018.

\subsection{RNA extraction and quantitative reverse-transcription polymerase chain reaction}

Total RNA was extracted from 4- $\mu$ m-thick FFPE specimens using the RNeasy FFPE Kit (Qiagen, Hilden, Germany). The complementary DNA (cDNA) synthesis was performed using PrimeScript RT Master Mix (RR036A) (Takara, Dalian, China). The quantitative reverse-transcription polymerase chain reaction (qRTPCR) assays were performed using the ViiA 7 Dx RT-PCR System (Applied Biosystems, Foster City, USA) with PowerUp SYBR Green Master Mix (Applied Biosystems, Vilnius, Lithuania). The expression of target genes was normalized with the housekeeping gene GAPDH using the 2- $\triangle \mathrm{CT}$ method. The primer sequences are provided in Table S2.

\subsection{Function enrichment analysis}

Metascape (https://metascape.org/) ${ }^{13}$, which is an online tool, was used to annotate the functional and pathway enrichment analyses to determine the potential molecular mechanisms and biological processes of the candidate genes. Metascape was used to perform Gene Ontology (GO) and The Kyoto Encyclopedia of Genes and Genomes (KEGG) pathway analyses for DEGs and IRDEGs. Select the most enriched set of genes in the cluster as one of the representative clusters.

\subsection{Gene set enrichment analysis and principal components analysis}


To determine the potential immune-related pathways and genes between TP53 ${ }^{\text {mut }}$ and TP53 ${ }^{\text {Wt }}$ patients with LUAD in the TCGA cohort, gene set enrichment analysis (GSEA) (version 4.0.0; https://www.gseamsigdb.org/gsea/index.jsp) was performed. An annotated gene set file (msigdb.v7.0.entrez.gmt) was selected for use as the reference gene set. Principal components analysis (PCA) was carried out using the "pca3d" R package (version 0.10.1; https://cran.r-project.org/web/packages/pca3d/) to investigate the relative infiltration patterns of high- and low-index patients.

\subsection{CIBERSORTX analysis}

The CIBERSORTx website (https://cibersort.stanford.edu/index.php) provides an algorithm to quantify the relative infiltration of several immune-infiltrating cells in the $\mathrm{TME}^{12}$, including $\mathrm{T}$ cell subtypes, naive and memory B cells, myeloid cell subsets, natural killer (NK) cells, and plasma cells. The data of immuneinfiltrating cell infiltration levels in patients with LUAD were extracted from CIBERSORTx to investigate the correlation with the IRM index.

\subsection{Immunohistochemistry}

FFPE LUAD specimens from the Nanjing cohort were serially sectioned at 4- $\mu$ m-thick spacings and then mounted on glass slides. In brief, we used ethylenediaminetetraacetic acid (EDTA) antigen retrieval buffer boiling to deparaffinize the sections and then incubated them with antibodies (anti-PD-1 antibody, 1:1000 dilution, Proteintech, Wuhan, China; anti-PD-L1 antibody, 1:2000 dilution, Proteintech, Wuhan, China) overnight at $4{ }^{\circ} \mathrm{C}$. The sections were subsequently analyzed using the HRP-Polymer anti-Mouse/Rabbit IHC Kit (MaxVision, Fuzhou, China). Immunohistochemistry (IHC) staining results were assessed and scored independently by 2 senior pathologists. The expression of PD-1 and PD-L1 in the tumor was determined as the proportion of positively colored cells compared to the total number of cells counted.

\subsection{Nomogram construction and validation}

Univariate and multivariate Cox analyses were used to evaluate the IRM index as an independent prognostic factor. Then, a concise nomogram to predict the OS of LUAD was established using the "rms" R package (version 2.10; https://cran.r-project.org/web/packages/rsm/index.html), including 4 factors. We conducted 5- and 7-year OS alignments to determine the prognostic ability of the nomogram model.

\subsection{Statistical analysis}

Statistical analyses were performed using R (version 3.6.1). The Student's t-test was used to determine differences for 2-group comparisons.

\section{Results}

\subsection{Immune landscape based on TP53 mutations in LUAD}


In LUAD, TP53 mutation is the most widespread somatic nonsynonymous mutation (Fig. 1A). To comprehensively evaluate the correlation between immune status and TP53 mutation in patients with LUAD, a flowchart was constructed to reveal the analyzing process (Fig. 1B). In the TCGA cohort, LUAD

samples were divided into 2 groups: TP53 ${ }^{\text {WT }}$ (260 patients) and TP53 ${ }^{\text {MUT }}$ (239 patients). Subsequently, we performed DEG analysis based on TP53 mutations, which revealed that 1829 genes were statistically significantly differentially expressed in the TP53 ${ }^{\mathrm{MUT}}$ group compared with the TP53 ${ }^{\mathrm{WT}}$ group, including 1230 downregulated genes and 599 upregulated genes (Fig. S1A, Table S3).

Metascape was used to annotate potential functional characteristics, and it showed that the DEGs mentioned above were mainly involved in pathways related to immunology (Fig. S1B). Next, to further prove the correlation between DEGs and tumor immunology, GSEA was used to show that TP53 ${ }^{\text {wt }}$ patients with LUAD were enriched in 324 biological processes, including 2 immune-related functional pathways: GO_REGULATION_OF_HUMORAL_IMMUNE_RESPONSE and GO_INNATE_IMMUNE_ RESPONSE_ACTIVATING_CELL_SURFACE_RECEPTOR_SIGNALING_PATHWAY (Fig. 1C), which further confirmed the potential correlation between immunology and TP53 status.

\subsection{IRM index construction and evaluation of its prognostic ability}

To determine the relationship between TP53 mutation status and immune-related functional pathways, 75 IRDEGs were selected for further analysis from the 1829 DEGs based on the Immport database (Table S4). PCA indicated that these 75 IRDEGs could distinguish between the TP53 ${ }^{\text {WT }}$ and TP53 ${ }^{\text {MUT }}$ groups (Fig. 2A). Next, we attempted to evaluate the prognostic performance of the 75 IRDEGs. Six genes with non-zero regression coefficients, including CRHR2, BPIFB2, INHA, SSTR5, SCGB3A1, and BPIFB1, were found to have a maximum prognostic value according to LASSO and Cox regression analysis (Fig. 2B, 2C). Ultimately, a 6-gene IRM was built, and the risk index of each patient was calculated using the following formula for further analysis: IRM index $=(-0.121586693 \times C R H R 2$ expression $)+(-0.048377824$ $\times$ BPIFB2 expression $)+(0.045865315 \times$ INHA expression $)+(-0.018472910 \times$ SSTR5 expression $)+$ $(-0.011651852 \times S C G B 3 A 1$ expression $)+(-0.002185301 \times$ BPIFB1 expression) (Fig. 2D). The optimal cutpoint was -0.9595243 . We annotated the immune-related pathways enriched by the 6 IRM-related genes, which included transforming growth factor (TGF) family members, cytokines, cytokine receptors, and antimicrobials (Fig. 2E). Cancer cells and several immune-suppressive cells in the TME recruit multiple cytokines, such as TGF- $\beta$, which facilitates tumor progression and mediates $T$ cell dysfunction ${ }^{14}$. This suggests that our IRM may be related to the immunosuppressive state of LUAD, which we will study next.

The cut-point in the TCGA cohort was used to assign patients with LUAD to the high- or low-index groups across all 3 cohorts. The Kaplan-Meier analysis illustrated that a high index was related to worse prognosis (Fig. 3A). The IRM index distribution, OS, and 6-gene expression heatmap are shown in Fig. 3B. The time-dependent ROC curve analysis of the IRM index in the TCGA cohort revealed the robustness of the prognostic capability of the IRM index for OS ( 3 years, $A U C=0.717 ; 5$ years, AUC $=0.730 ; 7$ years, AUC $=0.731$; Fig. 3C). 


\subsection{Validation of the predictive ability of the IRM in the meta-GEO and Nanjing cohorts}

To identify the robustness of the IRM index, its capability was evaluated in an independent validation cohort (the meta-GEO cohort), which included 131 patients with LUAD. Using the same formula and cutpoint as in the TCGA cohort, the patients in the meta-GEO cohort were divided into high- and low-index groups. The results revealed that high-index patients had outstandingly worse OS compared to patients with a low index, which was consistent with the results of the TCGA cohort (Fig. 3D). The IRM index distribution, OS, and 6-gene expression heatmap are shown in Fig. 3E. The time-dependent ROC curve analysis of the IRM index in the meta-GEO cohort revealed the robustness of the prognostic capability of the IRM index for OS ( 3 years, AUC $=0.643 ; 5$ years, $A U C=0.635 ; 7$ years, $A U C=0.620$; Fig. 3F). To better validate the clinical value of the IRM index, we assessed its prognostic capability in the Nanjing cohort, which included 92 patients who were diagnosed with LUAD at the Jiangsu Cancer Hospital and underwent radical surgery without neoadjuvant chemotherapy. The patients in the Nanjing cohort were divided into high- and low-index groups. The Kaplan-Meier analysis revealed consistent results in the Nanjing cohort (Fig. 3G). The IRM index distribution, OS, and 6-gene expression heatmap are shown in Fig. $3 \mathrm{H}$. The time-dependent ROC curve analysis of the Nanjing cohort revealed the robustness of the prognostic capability of the IRM index for OS ( 3 years, AUC $=0.705 ; 5$ years, AUC $=0.608 ; 7$ years, AUC $=$ 0.609; Fig. 3I).

\subsection{Validation of the IRM in different clinical subgroups}

TP53 status has a remarkable relationship with the clinical outcome of patients with LUAD. Stratification analyses were used to test the prediction ability of the IRM index in the TP53 ${ }^{\text {mut }}$ and TP5 $3^{\text {Wt }}$ subgroups. As shown in Fig. 4A and 4B, both in the TP53 ${ }^{\text {mut }}$ and TP53 ${ }^{\text {wt }}$ subgroups, patients with a high index had worse OS than patients with a low index. Apart from TP53 status, factors such as age, sex, and tumornode-metastasis (TNM) stage may also affect LUAD prognosis. We illustrated that the IRM index was a robust biomarker for estimating OS in younger ( $\leq 65$ years) or older ( $>65$ years), male or female, and early tumor stage (TNM stage I) or advanced tumor stage (TNM stage II, III, and IV) patients (Fig. 4C-4H).

\subsection{Immune status between the low- and high-index patients with LUAD}

Characterization of the immune infiltration landscape is important to further explain the potential correlation between the IRM index and tumor humoral immunology by the status of the immune microenvironment. First, using Metascape, we annotated the functions of the 75 IRDEGs, which overlapped with the DEGs and Immport database. These IRDEGs were mainly enriched in terms of antimicrobial humoral response, hormone level regulation, hormone secretion, and neuroactive ligand binding (Fig. S2A-S2C). This data thus provided solid evidence of the potential relationship between the IRM index and tumor humoral immunology. Meanwhile, the results prompted us to further explore the correlation between the IRM and immune-related biological processes. Using CIBERSORTX, we identified 
the relationships between the IRM index and 22 infiltrating immune cells, which were acquired in the TCGA cohort (Fig. 5A). High- and low-index patients were segmented into 2 distinct clusters using PCA based on the relative infiltration of the above-mentioned cell subpopulations (Fig. 5B). Additionally, the relative infiltration of the 22 immune-infiltrating cells showed mild to moderate association (Fig. S3). The high-risk patients with LUAD had remarkably lower relative infiltration proportions of memory $B$ cells and regulatory T cells (Tregs; Fig. 5C, 5D) and significantly higher relative infiltration proportions of neutrophils and resting memory CD $4+T$ cells (Fig. 5E, 5F) than low-risk patients with LUAD. Thus, the above results suggested that the heterogeneity of tumor immune-related cell infiltration in LUAD may be a potential prognostic biomarker and target for response after immunotherapy and may have remarkable clinical significance.

Furthermore, immune checkpoint inhibitors play an antitumor role, reversing the immunosuppressive effect of the tumor. We investigated the correlation between the IRM index and the expression of crucial immune checkpoints, including PDCD1 (encoding PD-1), LAG3, TIGIT, TIM3, CD274 (encoding PD-L1), and CTLA4. The correlation coefficient is shown in Table S5. We observed that the IRM index had a remarkably positive relationship to the expression of $T I M 3, T I G I T, P D C D 1$, and $C D 274$ ( $\mathrm{P}<0.05$; Fig. $6 \mathrm{~A}$ ). In addition, the expression of $P D C D 1$ and $C D 274$ in the high-index group was remarkably higher than in the low-index group (Fig. 6B, 6C). To validate the expression differences in PDCD1 and CD274 between the high- and low- index patients, we detected the PDCD1 and CD274 expression in the Nanjing cohort, and the high-index patients expressed higher levels of PDCD1 and CD274 (Fig. 6D, 6E). These results are consistent with a previous study showing that PD-L1 expression is related to advanced pathological features and worse OS in patients with NSCLC ${ }^{15}$. We also found the expression differences of PD-1 and PD-L1, which are encoded by PDCD1 and CD274, respectively, using IHC samples from 12 patients in the same cohort. These results revealed a significantly positive correlation between the IRM index and IHC scores of these 2 immune checkpoint proteins in patients with LUAD (Fig. 6F-6H).

\subsection{Development and verification of a nomogram based on the IRM index}

To assess the prognostic value of the IRM index, a Cox regression analysis was conducted in the TCGA cohort. We first selected the IRM index and several clinical factors for univariate Cox analysis. As shown in Fig. 7A, factors including lymphatic invasion, distant metastasis, IRM index, age, and TNM stage were significantly related to OS in the TCGA cohort $(P<0.05)$. After multivariate Cox regression analysis of these factors, lymphatic invasion, IRM index, age, and TNM stage were still significantly associated with survival $(P<0.05)$. Based on these results, we further integrated the IRM and multiple independent clinical factors (lymphatic invasion, age, and TNM stage) to develop a nomogram, which gives clinicians a quantitative way to predict the clinical outcome of patients with LUAD (Fig. 7B). The C-index for the nomogram in the TCGA cohort was 0.746 (95\% Cl: 0.5886-0.8730). Calibration plots comparing the predicted and actual outcomes of 5- and 7-year OS indicated good agreement (Fig. 7C, 7D). Additionally, we used a time-dependent ROC curve analysis to compare the robustness of the prognostic capability 
between the nomogram, IRM index, age, and TNM stage in 5- and 7-year OS (Fig. 7E, 7F). The nomogram revealed higher prognostic capability at 5 and 7 years with a larger AUC. Furthermore, we verified the prognostic accuracy in the Nanjing cohort. As we expected, the nomogram obtained consistent results (Fig. 7G, 7H).

\section{Discussion}

In this study, we systematically analyzed the association between TP53 status and immune-related phenotypes in patients with LUAD. A TP53-related IRM index was constructed. It was derived from the TCGA cohort and validated in the meta-GEO and Nanjing cohorts. It was found to be remarkably associated with prognosis. The prognostic value of the 6-gene IRM index was independent of multiple known strong prognostic factors. Furthermore, the IRM index allowed us to divide patients with LUAD into 2 subgroups with different immune-related phenotypes. Consequently, we integrated the IRM with multiple clinical factors into a nomogram with robust OS prediction.

Through multi-functional enrichment analysis of DEGs, we observed obvious enrichment in the humoral immune response. The consistent pathway enrichment analysis results were further verified in selected IRDEGs. Furthermore, 6 IRM-related genes were found to participate in multiple immunosuppressionrelated pathways. Afterward, we divided the patients with LUAD into 2 subgroups with different prognosis and immune-related phenotypes. In all 3 cohorts, high-index patients showed worse survival than lowindex patients. Meanwhile, the IRM index was positively correlated with the expression of several important immune checkpoints. In previous research, NSCLC progression was positively associated with the increased expression of T cell exhaustion markers, such as PD-1, TIM3, and CTLA4 ${ }^{16}$, which is consistent with the results of our research.

CRHR2 belongs to the G-protein coupled receptor superfamily, regulating corticotropin-releasing hormone to perform biological functions ${ }^{17}$, which widely expressed in the gastrointestinal tract, lung and skeletal muscle $^{18}$. CRHR2 stimulates intracellular cAMP pathway, including activation of nuclear factor-kB and expression of TNF- $\beta$ in T cells ${ }^{19,20}$. BPIFB1 and BPIFB2 belong to the bactericidal/permeabilityincreasing-fold-containing family ${ }^{21}$. BPIFB1 and BPIFB2 protein are most highly expressed in the trachea and lung ${ }^{22}$ and bind to the Gram-negative bacteria and exert antibacterial function ${ }^{23,24}$. Moreover, it has been reported that BPIFB1 is abnormally expressed in tumors, which suggests that it plays a role in tumor development ${ }^{25}$. INHA encodes a member of the TGF- $\beta$ superfamily of proteins (RefSeq, Aug 2016), which perform the functions of activated cytokine and hormone 26,27 . SSTR5 is a predominant component of somatostatin receptor subtypes, which regulates inhibitory effects on endocrine and exocrine secretions ${ }^{28}$. SSTR5 has a significant regulating-inflammatory effect via regulating somatostatin, which demonstrated in different animal models ${ }^{29,30}$. SCGB3A1 belong to secretoglobin gene superfamily, which are cytokine-like small molecular weight secreted proteins and predominantly expressed in lung airway epithelial cells ${ }^{31}$. Secretoglobins are thought to be involved in immunomodulatory ${ }^{32}$. Moreover, SCGB3A1 
has been reported as a tumor suppressor in various human tumors including breast, prostate, lung, and pancreatic carcinomas ${ }^{33,34}$.

In chronic diseases, T cells malfunction due to T cell exhaustion, which increases the expression of inhibitory receptors incorporating PD-1, LAG3, TIM3, CTLA4, and TIGIT ${ }^{35-39}$, resulting in fewer cytokines and loss of antitumor capabilities. The limited efficacy of immunotherapy may be due to the production of dysfunctional $\mathrm{T}$ cells in the $\mathrm{TME}^{40}$. Therefore, regulators that reverse the state of $\mathrm{T}$ cell dysfunction are the focus of current research. For example, tertiary lymphoid structure immune activity dysfunction is reversed and antitumor capabilities are enhanced after treatment with anti-PD-1/PD-L1 and anti-CTLA-4 immune checkpoint inhibitors in humans and mice ${ }^{41-44}$. The PD-L1/PD-1 axis is an important regulatory pathway of T cell exhaustion in tumors. DD1a expression induced by p53, which is encoded by TP53, has been shown to upregulate PD-1 and PD-L1, as cancer cells respond to genotoxic stress and DNA damage, which then promotes the gradual priming of immune surveillance ${ }^{45}$. This finding clarified the relationship between p53 and immune checkpoint inhibitors, which may indicate the intrinsic molecular mechanism of the relationship between the IRM index and the expression of immune checkpoints. PD-L1 has abundant expression in cancer cells and the tumor extracellular matrix, and blocking the PD-L1/PD-1 axis can enhance the antitumor capabilities of $\mathrm{T}_{\text {cells }}{ }^{46}$. In our study, the IRM index was positively correlated with the expression of immune checkpoints, which shows that as the IRM index grows, T cells are exhausted, and their antitumor abilities decrease. This explains the poor prognosis of high-index patients with LUAD. It can be speculated that immunotherapies that block the pathways that suppress tumor immune responses for patients with LUAD in the high-index group may increase the presentation of cancer-associated antigens, resulting in the recovery of the immune response of CD $8+T$ cells ${ }^{47}$, which may then result in better immunotherapy effects.

In the immune infiltration analysis between the high- and low-index patients, the high-index patients with LUAD had remarkably higher proportions of neutrophils and resting memory CD4 + T cells and lower proportions of memory B cells and Tregs. Memory resting CD4 + T cells can be further differentiated into multiple cell subpopulations and confer different functions, including blocking CD8 $+\mathrm{T}$ cell activation and NK cell killing and suppressing the immune response to autoantigens and exogenous antigens ${ }^{48}$. In human NSCLC, neutrophils play a key role in tumor immunity ${ }^{49}$. In mice, neutrophils infiltrating tumors can either promote carcinogenesis by supporting tumor-related inflammation, angiogenesis, and metastasis and inhibiting $T$ cell activation or restrict tumor growth through the expression of antitumor and cytotoxic mediators ${ }^{50}$. Nevertheless, because the tumor is in an irreversible continuous chronic inflammatory state, suppressive neutrophils are constantly mobilized and become the dominant subpopulation of neutrophils ${ }^{51}$, which may suppress the immune response and promote malignant progression. In our study, the high-index patients with LUAD had significantly higher proportions of neutrophils and resting memory CD4 + T cells, restricting the function of CD $8+T$ cells and NK cells in the tumor development process, resulting in malignant tumor progression and worse OS. It is reasonable to speculate that the high-index patients with LUAD may have improved CD $8+T$ cell function and receive better efficacy by using an immune checkpoint inhibitor that blocks the PD-1/PD-L1 axis. 
Tregs are an inhibitory subpopulation of CD $4+T$ cells. Cancer-associated fibroblasts in the extracellular matrix express COX-2, which promotes PGE2 secretion to induce immunosuppressive FOXP3 + Tregs ${ }^{52}$, which then accumulate in primary tumor tissues and the peripheral blood to promote immune evasion ${ }^{46}$. Progressing tumors can inhibit CD8 $+T$ cells through several approaches, including Tregs, which can directly suppress the antitumor functions of $\mathrm{CD} 8+T$ cells $^{53}$. The immunosuppressive state of the highindex patients with LUAD may not through the suppression of $C D 8+T$ cells immune response by Tregs. Tumor-infiltrating B cells are a key component of the TME. B cells and CD $4+T$ cells together form tertiary lymphoid structures, which are related to better outcomes ${ }^{54}$. This is consistent with the results of our study, as low-index patients had better prognoses. A variety of cytokines, including tumor necrosis factor (TNF), interleukin (IL)-2, IL-6, and interferon gamma (IFNY), which are secreted by $B$ cells, could assist $B$ cells to recruit other immune effector cells and activate immune responses, including $T$ cells. Memory $B$ cells may play a role in inhibiting antigen presentation, thereby driving the expansion of memory and naive T cell responses. Memory B cells and plasma cells show similar characteristics of facilitating an acquired immune response, which also contributes to an effective $T$ cell response after immune checkpoint inhibitor therapy ${ }^{55}$. The high-index patients with LUAD had lower proportions of memory $B$ cells, indicating that the suppression of T cell antitumor functions in such patients may be due to the decline of memory $B$ cell antigen presentation functions, which is related to worse OS.

Our research supplies a novel angle regarding LUAD immune microenvironment groupings and immunotherapy responses. However, since our study is a retrospective study, it has limitations and needs to be further validated by prospective studies. Furthermore, mechanistic studies of the IRM index-related genes and immune-infiltrating cells need to be implemented to explain their clinical application. In subsequent research, we will focus on single-cell transcriptome studies of immune-infiltrating cells in patients with LUAD with different IRM indexes.

In conclusion, the IRM index is a robust clinical biomarker that can assign patients with LUAD to subgroups with significantly different prognoses and immune-related phenotypes, which may explain the molecular mechanism of different prognoses from the perspective of immunology.

\section{Abbreviations}

\section{LUAD}

Lung adenocarcinoma

NSCLC

Non-small cell lung cancer

TME

tumor micro-environment

IRM

immune-related model

DEGs 
differentially expressed genes

\section{Declarations}

\section{Data accessibility statement}

The data sets supporting the conclusions of this article are available from the "TCGAbiolinks" R package (version 2.14.1; https://bioconductor.org/packages/release/bioc/html/TCGAbiolinks.html) and GEO database (https://www.ncbi.nlm.nih.gov/gds).

\section{Ethics approval and consent to participate}

This study was approved by the Regional Ethics Committee at Nanjing Medical University. The experiments were undertaken with the understanding and written consent of each patient. The methodologies conformed to the standards set by the Declaration of Helsinki.

\section{Consent for publication:}

The consent was obtained from patients.

\section{Conflict of interest}

The authors declare that the research was conducted in the absence of any commercial or financial relationships that could role as a potential conflict of interest.

\section{Author contributions}

Xuming Song, Jifan Wang and Qiang Chen conceived and designed the experiments. Xuming Song and Jifan Wang performed the analysis. Xuming Song and Qiang Chenwrote the paper. Qixing Mao helped with the statistical analysis. Lin Xu, Feng Jiang, Gaochao Dong, Wenjie Xia reviewed the draft. All authors read and approved the final manuscript.

\section{Acknowledgements}

We would like to thank Dr. Dan Chen and Dr. Yingkuan Liang for helpful discussions and suggestions.

\section{Funding}

This study was supported by the grants from the National Natural Science Foundation of China (Grant No. 81672294, 81702892); The Project of Invigorating Health Care through Science $\triangle T$ Technology Education, Jiangsu Provincial Medical Innovation Team (CXTDA2017002); Funded by Jiangsu Provincial key research development program (BE2017761);The Foundation of Jiangsu Cancer hospital (ZK201601) ;The young talents program of Jiangsu Cancer Hospital (23). 


\section{References}

1. Travis WD, Brambilla E, Nicholson AG, et al. The 2015 World Health Organization Classification of Lung Tumors: Impact of Genetic, Clinical and Radiologic Advances Since the 2004 Classification. J Thorac Oncol. 2015;10(9):1243-60.

2. Tang H, Qiao J, Fu YX. Immunotherapy and tumor microenvironment. Cancer Lett. 2016;370(1):8590.

3. Vokes EE, Ready N, Felip E, et al. Nivolumab versus docetaxel in previously treated advanced nonsmall-cell lung cancer (CheckMate 017 and CheckMate 057): 3-year update and outcomes in patients with liver metastases. Ann Oncol. 2018;29(4):959-65.

4. Borghaei H, Paz-Ares L, Horn L, et al. Nivolumab versus Docetaxel in Advanced Nonsquamous NonSmall-Cell Lung Cancer. N Engl J Med. 2015;373(17):1627-39.

5. Horn L, Spigel DR, Vokes EE, et al. Nivolumab Versus Docetaxel in Previously Treated Patients With Advanced Non-Small-Cell Lung Cancer: Two-Year Outcomes From Two Randomized, Open-Label, Phase III Trials (CheckMate 017 and CheckMate 057). J Clin Oncol. 2017;35(35):3924-33.

6. Maddocks OD, Berkers CR, Mason SM, et al. Serine starvation induces stress and p53-dependent metabolic remodelling in cancer cells. Nature. 2013;493(7433):542-6.

7. Jones RG, Plas DR, Kubek S, et al. AMP-activated protein kinase induces a p53-dependent metabolic checkpoint. Mol Cell. 2005;18(3):283-93.

8. Berkers CR, Maddocks OD, Cheung EC, Mor I, Vousden KH. Metabolic regulation by p53 family members. Cell Metab. 2013;18(5):617-33.

9. Levine AJ, Tomasini R, McKeon FD, Mak TW, Melino G. The p53 family: guardians of maternal reproduction. Nat Rev Mol Cell Biol. 2011;12(4):259-65.

10. Vousden KH, Ryan KM. p53 and metabolism. Nat Rev Cancer. 2009;9(10):691-700.

11. Dong ZY, Zhong WZ, Zhang XC, et al. Potential Predictive Value of TP53 and KRAS Mutation Status for Response to PD-1 Blockade Immunotherapy in Lung Adenocarcinoma. Clin Cancer Res. 2017;23(12):3012-24.

12. Newman AM, Steen CB, Liu CL, et al. Determining cell type abundance and expression from bulk tissues with digital cytometry. Nat Biotechnol. 2019;37(7):773-82.

13. Zhou Y, Zhou B, Pache L, et al. Metascape provides a biologist-oriented resource for the analysis of systems-level datasets. Nat Commun. 2019;10(1):1523.

14. Zhang Z, Liu S, Zhang B, Qiao L, Zhang Y, Zhang Y. T Cell Dysfunction and Exhaustion in Cancer. Front Cell Dev Biol. 2020;8:17.

15. Yamamoto R, Nishikori M, Kitawaki T, et al. PD-1-PD-1 ligand interaction contributes to immunosuppressive microenvironment of Hodgkin lymphoma. Blood. 2008;111(6):3220-4.

16. Thommen DS, Schreiner J, Muller P, et al. Progression of Lung Cancer Is Associated with Increased Dysfunction of T Cells Defined by Coexpression of Multiple Inhibitory Receptors. Cancer Immunol Res. 2015;3(12):1344-55. 
17. Agelaki S, Tsatsanis C, Gravanis A, Margioris AN. Corticotropin-releasing hormone augments proinflammatory cytokine production from macrophages in vitro and in lipopolysaccharide-induced endotoxin shock in mice. Infect Immun. 2002;70(11):6068-74.

18. Chalmers DT, Lovenberg TW, Grigoriadis DE, Behan DP, De Souza EB. Corticotrophin-releasing factor receptors: from molecular biology to drug design. Trends Pharmacol Sci. 1996;17(4):166-72.

19. Zhao J, Karalis KP. Regulation of nuclear factor-kappaB by corticotropin-releasing hormone in mouse thymocytes. Mol Endocrinol. 2002;16(11):2561-70.

20. van der Meer MJ, Hermus AR, Pesman GJ, Sweep CG. Effects of cytokines on pituitary betaendorphin and adrenal corticosterone release in vitro. Cytokine. 1996;8(3):238-47.

21. Wei $F$, Tang L, He Y, et al. BPIFB1 (LPLUNC1) inhibits radioresistance in nasopharyngeal carcinoma by inhibiting VTN expression. Cell Death Dis. 2018;9(4):432.

22. Bingle CD, Craven CJ. PLUNC: a novel family of candidate host defence proteins expressed in the upper airways and nasopharynx. Hum Mol Genet. 2002;11(8):937-43.

23. Shao Y, Li C, Che Z, et al. Cloning and characterization of two lipopolysaccharide-binding protein/bactericidal permeability-increasing protein (LBP/BPI) genes from the sea cucumber Apostichopus japonicus with diversified function in modulating ROS production. Dev Comp Immunol. 2015;52(1):88-97.

24. Nam BH, Moon JY, Park EH, et al. Antimicrobial activity of peptides derived from olive flounder lipopolysaccharide binding protein/bactericidal permeability-increasing protein (LBP/BPI). Mar Drugs. 2014;12(10):5240-57.

25. Li J, Xu P, Wang L, et al. Molecular biology of BPIFB1 and its advances in disease. Ann Transl Med. 2020;8(10):651.

26. Shav-Tal Y, Zipori D. The role of activin a in regulation of hemopoiesis. Stem Cells. 2002;20(6):493500 .

27. Gaudet P, Livstone MS, Lewis SE, Thomas PD. Phylogenetic-based propagation of functional annotations within the Gene Ontology consortium. Brief Bioinform. 2011;12(5):449-62.

28. Ben-Shlomo A, Melmed S. Pituitary somatostatin receptor signaling. Trends Endocrinol Metab. 2010;21(3):123-33.

29. Helyes Z, Elekes K, Nemeth J, et al. Role of transient receptor potential vanilloid 1 receptors in endotoxin-induced airway inflammation in the mouse. Am J Physiol Lung Cell Mol Physiol. 2007;292(5):L1173-81.

30. Helyes Z, Pinter E, Sandor K, et al. Impaired defense mechanism against inflammation, hyperalgesia, and airway hyperreactivity in somatostatin 4 receptor gene-deleted mice. Proc Natl Acad Sci U S A. 2009;106(31):13088-93.

31. Jackson BC, Thompson DC, Wright MW, et al. Update of the human secretoglobin (SCGB) gene superfamily and an example of 'evolutionary bloom' of androgen-binding protein genes within the mouse Scgb gene superfamily. Hum Genomics. 2011;5(6):691-702. 
32. Naizhen X, Kido T, Yokoyama S, Linnoila RI, Kimura S. Spatiotemporal Expression of Three Secretoglobin Proteins, SCGB1A1, SCGB3A1, and SCGB3A2, in Mouse Airway Epithelia. J Histochem Cytochem. 2019;67(6):453-63.

33. Krop IE, Sgroi D, Porter DA, et al. HIN-1, a putative cytokine highly expressed in normal but not cancerous mammary epithelial cells. Proc Natl Acad Sci U S A. 2001;98(17):9796-801.

34. Krop I, Player A, Tablante A, et al. Frequent HIN-1 promoter methylation and lack of expression in multiple human tumor types. Mol Cancer Res. 2004;2(9):489-94.

35. Hashimoto M, Kamphorst AO, Im SJ, et al. CD8 T Cell Exhaustion in Chronic Infection and Cancer: Opportunities for Interventions. Annu Rev Med. 2018;69:301-18.

36. Jin HT, Anderson AC, Tan WG, et al. Cooperation of Tim-3 and PD-1 in CD8 T-cell exhaustion during chronic viral infection. Proc Natl Acad Sci U S A. 2010;107(33):14733-8.

37. Crawford A, Wherry EJ. The diversity of costimulatory and inhibitory receptor pathways and the regulation of antiviral T cell responses. Curr Opin Immunol. 2009;21(2):179-86.

38. Blackburn SD, Shin H, Haining WN, et al. Coregulation of CD8 + T cell exhaustion by multiple inhibitory receptors during chronic viral infection. Nat Immunol. 2009;10(1):29-37.

39. Joller N, Hafler JP, Brynedal B, et al. Cutting edge: TIGIT has T cell-intrinsic inhibitory functions. J Immunol. 2011;186(3):1338-42.

40. Thommen DS, Schumacher TN. T Cell Dysfunction in Cancer. Cancer Cell. 2018;33(4):547-62.

41. Wherry EJ, Kurachi M. Molecular and cellular insights into T cell exhaustion. Nat Rev Immunol. 2015;15(8):486-99.

42. Zarour HM. Reversing T-cell Dysfunction and Exhaustion in Cancer. Clin Cancer Res. 2016;22(8):1856-64.

43. Miller BC, Sen DR, Al Abosy R, et al. Subsets of exhausted CD8(+) T cells differentially mediate tumor control and respond to checkpoint blockade. Nat Immunol. 2019;20(3):326-36.

44. Wang D, Lin J, Yang X, et al. Combination regimens with PD-1/PD-L1 immune checkpoint inhibitors for gastrointestinal malignancies. J Hematol Oncol. 2019;12(1):42.

45. Yoon KW, Byun S, Kwon E, et al. Control of signaling-mediated clearance of apoptotic cells by the tumor suppressor p53. Science. 2015;349(6247):1261669.

46. Jiang Y, Li Y, Zhu B. T-cell exhaustion in the tumor microenvironment. Cell Death Dis. 2015;6:e1792.

47. Pardoll DM. The blockade of immune checkpoints in cancer immunotherapy. Nat Rev Cancer. 2012;12(4):252-64.

48. Rosenberg J, Huang J. CD8(+) T Cells and NK Cells: Parallel and Complementary Soldiers of Immunotherapy. Curr Opin Chem Eng. 2018;19:9-20.

49. Kargl J, Busch SE, Yang GH, et al. Neutrophils dominate the immune cell composition in non-small cell lung cancer. Nat Commun. 2017;8:14381.

50. Altorki NK, Markowitz GJ, Gao D, et al. The lung microenvironment: an important regulator of tumour growth and metastasis. Nat Rev Cancer. 2019;19(1):9-31. 
51. Granot Z. Neutrophils as a Therapeutic Target in Cancer. Front Immunol. 2019;10:1710.

52. Sharma S, Yang SC, Zhu L, et al. Tumor cyclooxygenase-2/prostaglandin E2-dependent promotion of FOXP3 expression and CD4 + CD $25+T$ regulatory cell activities in lung cancer. Cancer Res. 2005;65(12):5211-20.

53. Ganesan AP, Johansson M, Ruffell B, et al. Tumor-infiltrating regulatory T cells inhibit endogenous cytotoxic T cell responses to lung adenocarcinoma. J Immunol. 2013;191(4):2009-17.

54. Germain C, Gnjatic S, Tamzalit F, et al. Presence of B cells in tertiary lymphoid structures is associated with a protective immunity in patients with lung cancer. Am J Respir Crit Care Med. 2014;189(7):832-44.

55. Helmink BA, Reddy SM, Gao J, et al. B cells and tertiary lymphoid structures promote immunotherapy response. Nature. 2020;577(7791):549-55.

\section{Figures}


A

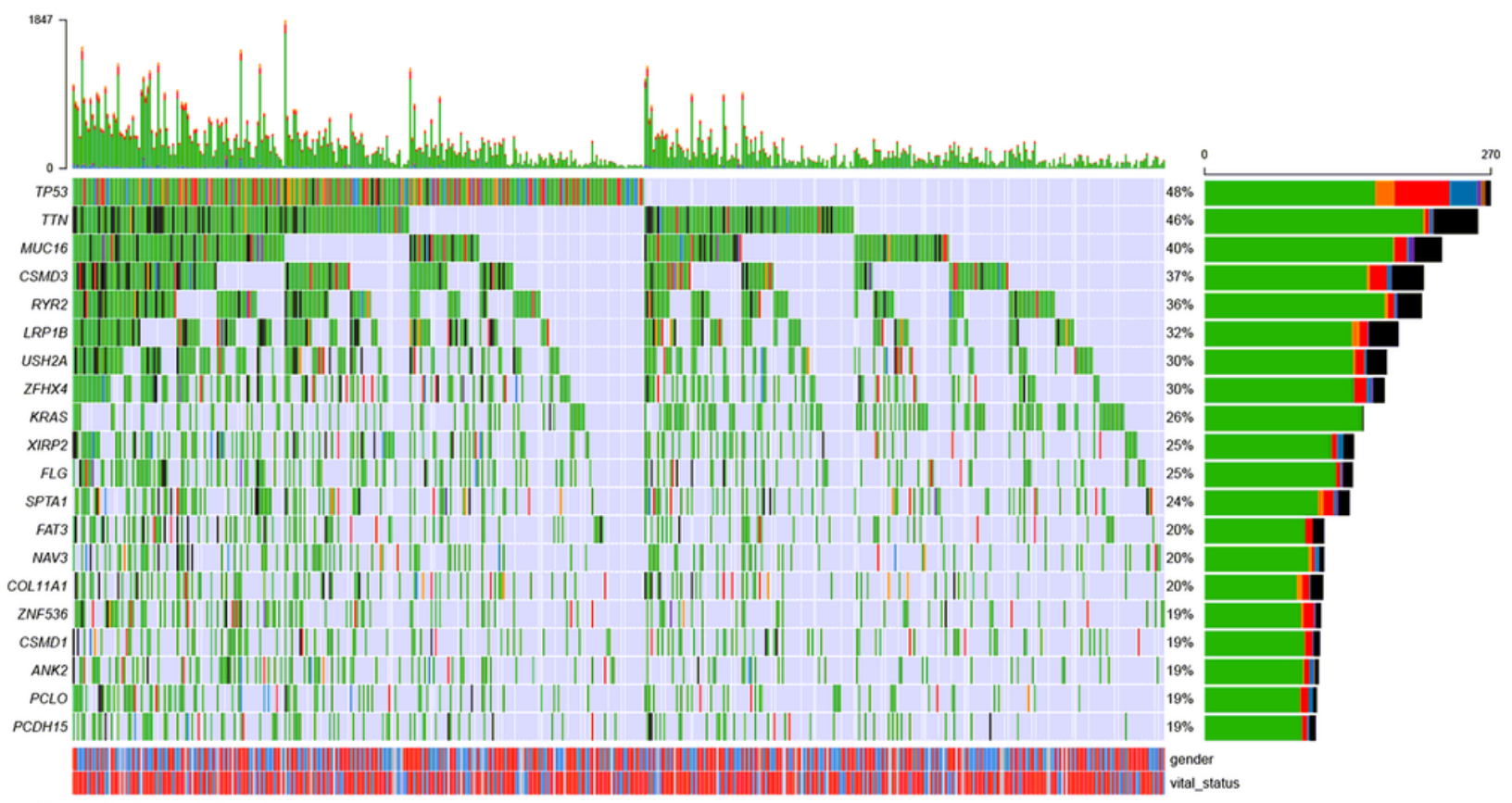

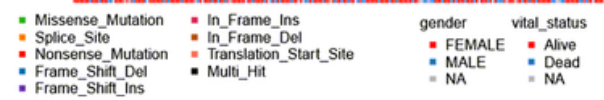

: Frame_Shif_Del

B

\section{Study design flow-chart}

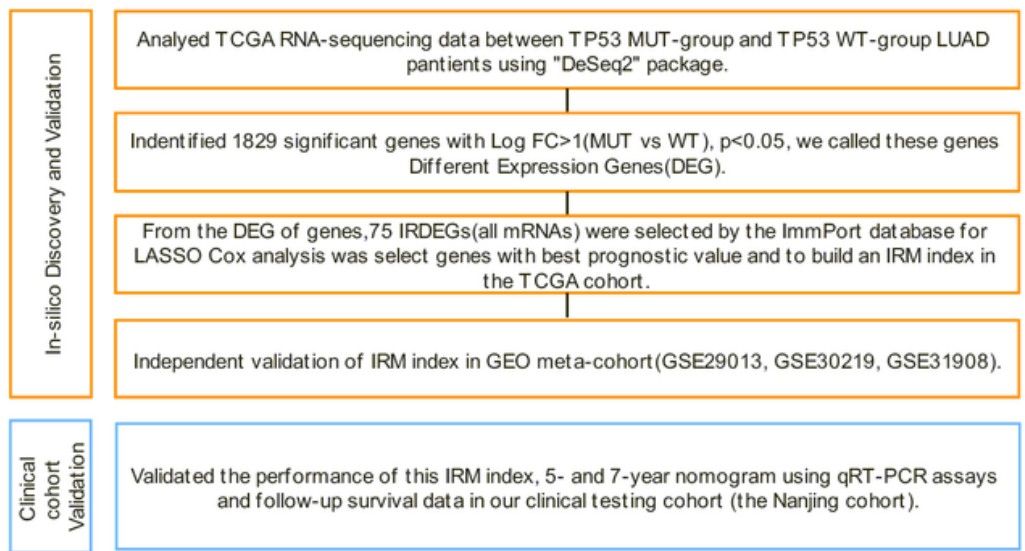

C

GO_REGULATION_OF_HUMORAL_IMMUNE_RESPONSE

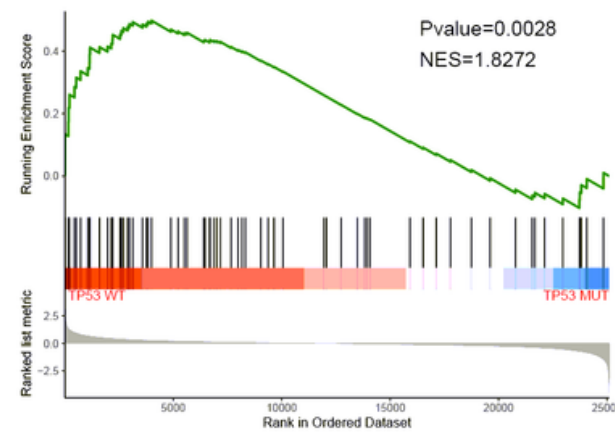

GO_INNATE_IMMUNE_RESPONSE_ACTIVATING_CELL_SURFACE_RECEPTOR_SIGNALING_PATHWAY

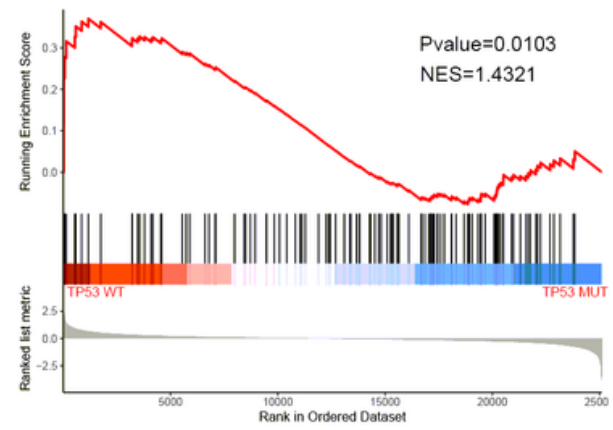

\section{Figure 1}

Study design and in silico discovery of TP53-associated genes. (A) Genomic landscape of lung adenocarcinoma and the mutational signature in the TCGA dataset. (B)The study design. (C) Significant enrichment of the immune-related phenotype in TP53MUT LUAD patients compared with that in TP53WT LUAD patients by GSEA. 

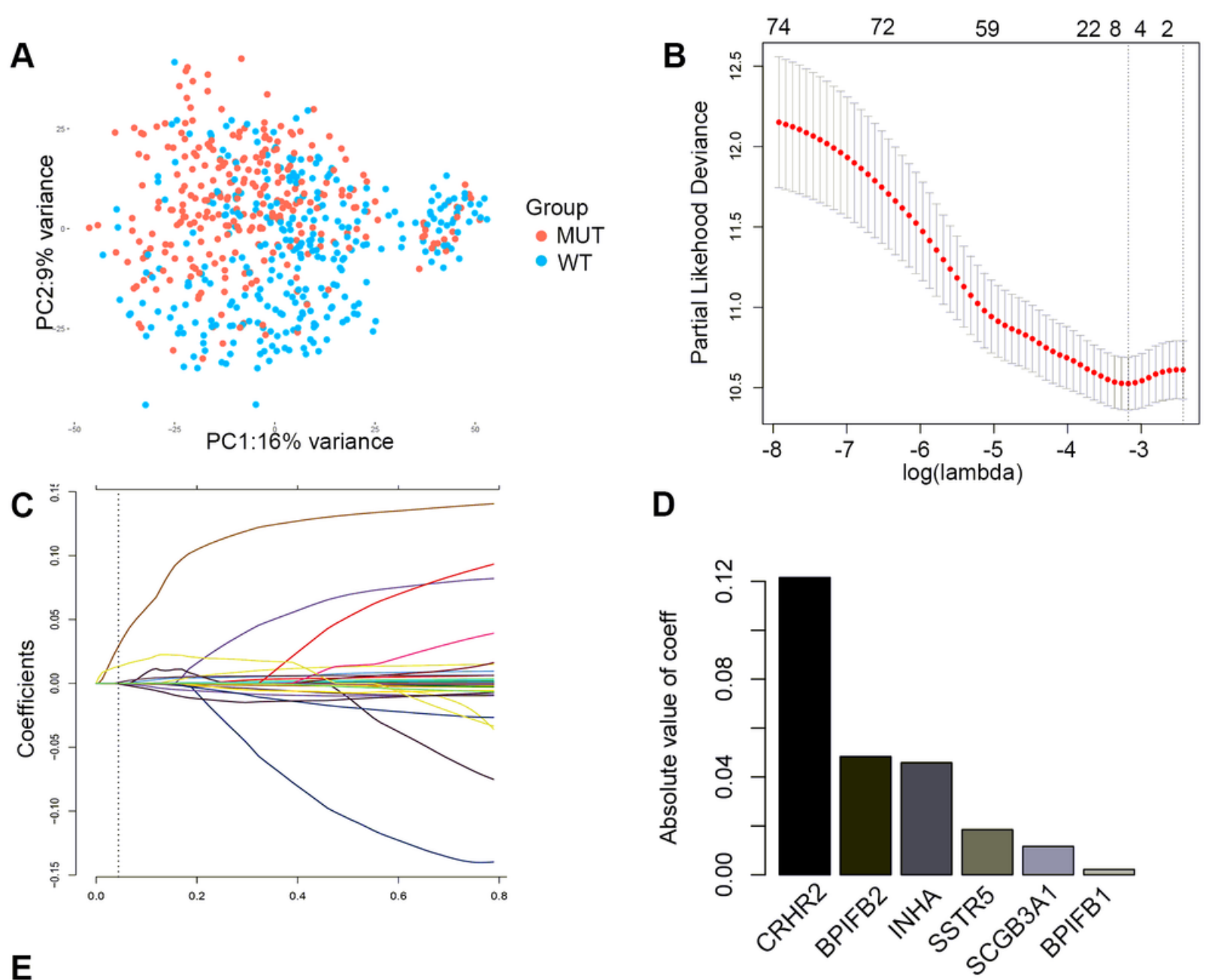

E

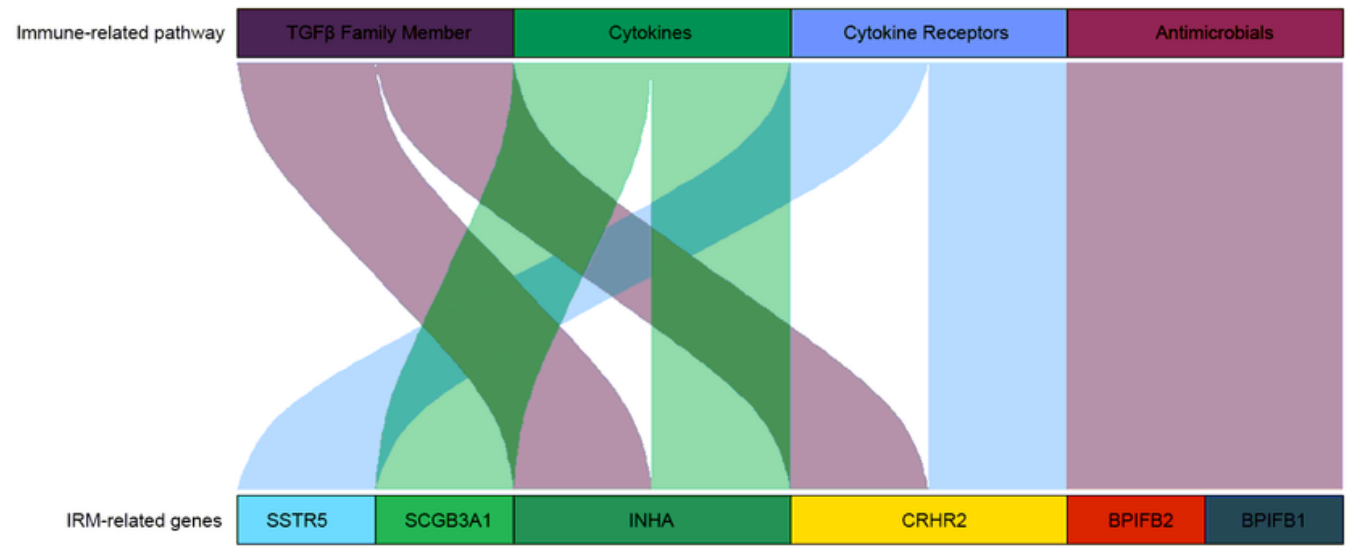

\section{Figure 2}

Identification of Immune-Related Models Index (IRM index). (A) Principal components analysis performed on lung adenocarcinoma patients based on significant differences immune-related RNA expressed between TP53MUT and TP53WT patients in the TCGA dataset. (B) Tuning parameter (lambda) screening in the LASSO regression model. (C) The LASSO coefficient profiles of the common genes. (D) Absolute value of coefficient for each of the six selected genes. (E) The immune-related pathways enriched by IRMrelated six genes. 


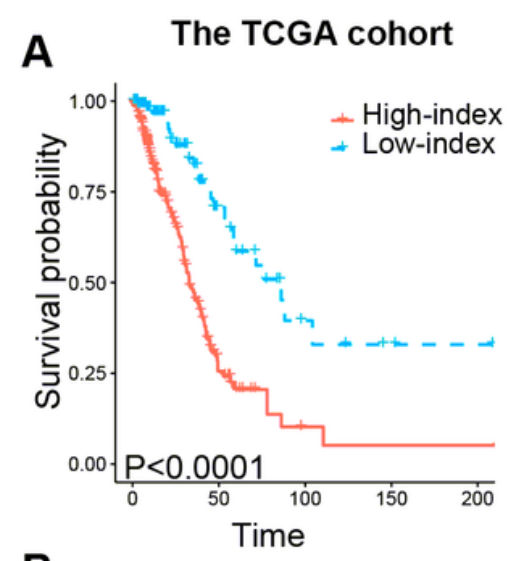

B
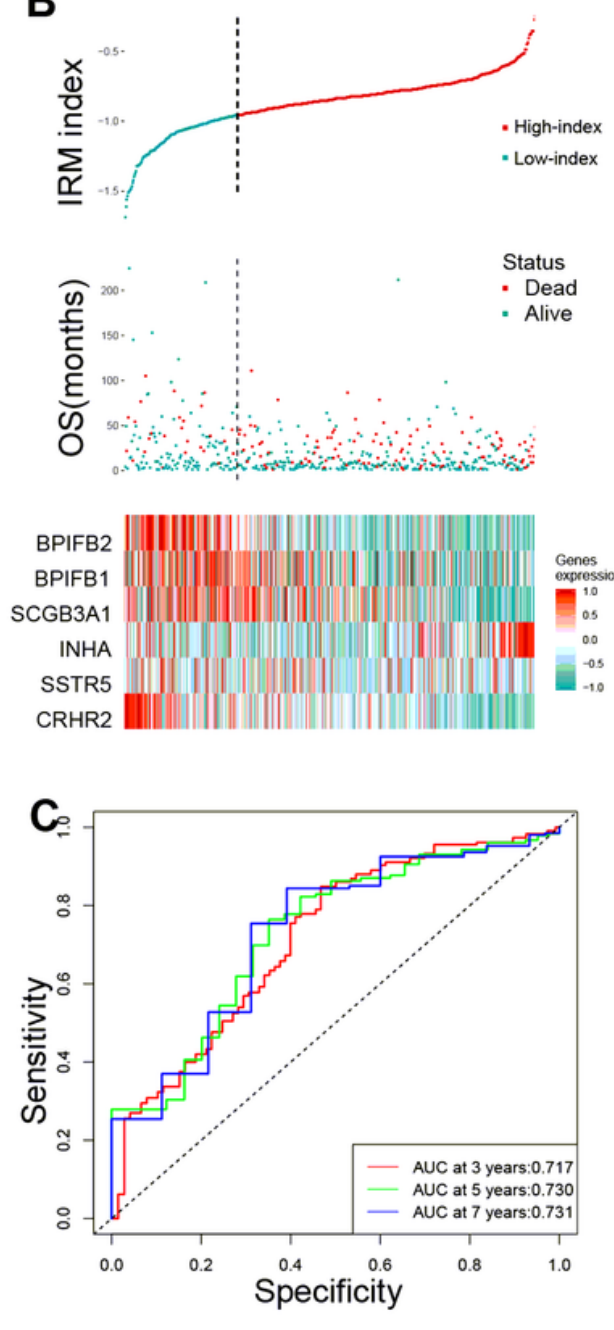

D

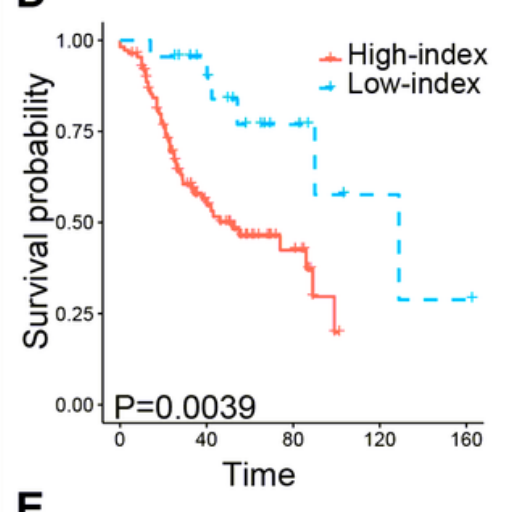

$\mathbf{E}$
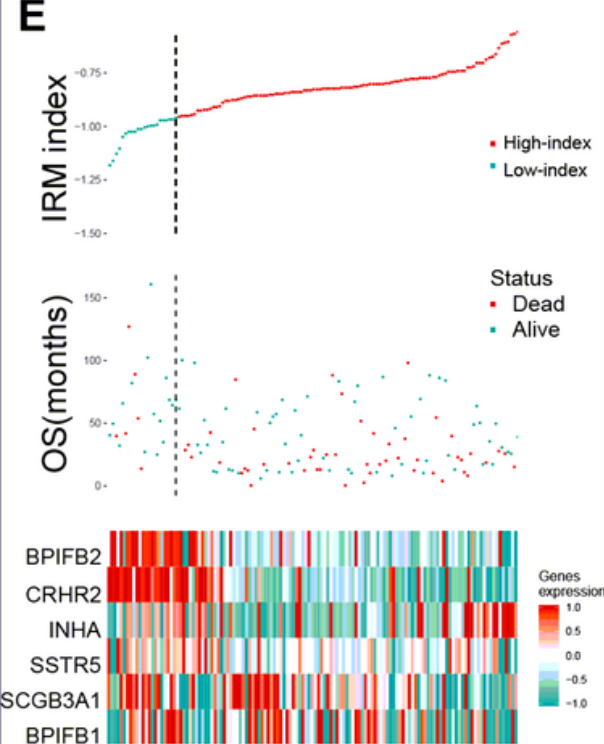
BPIFB

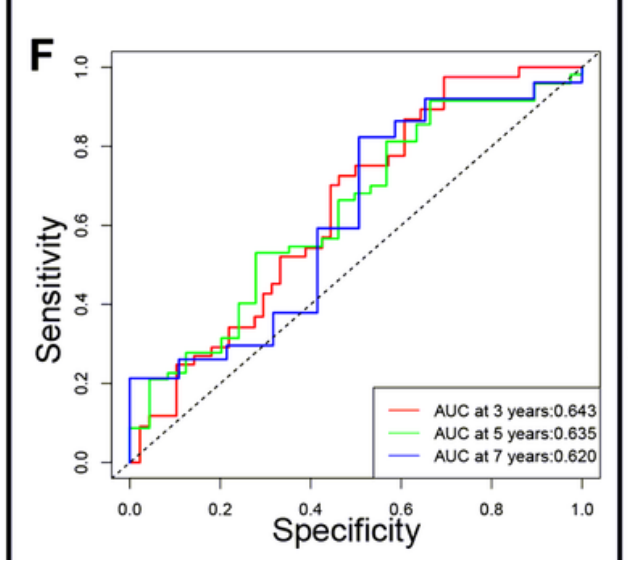

The Nanjing cohort
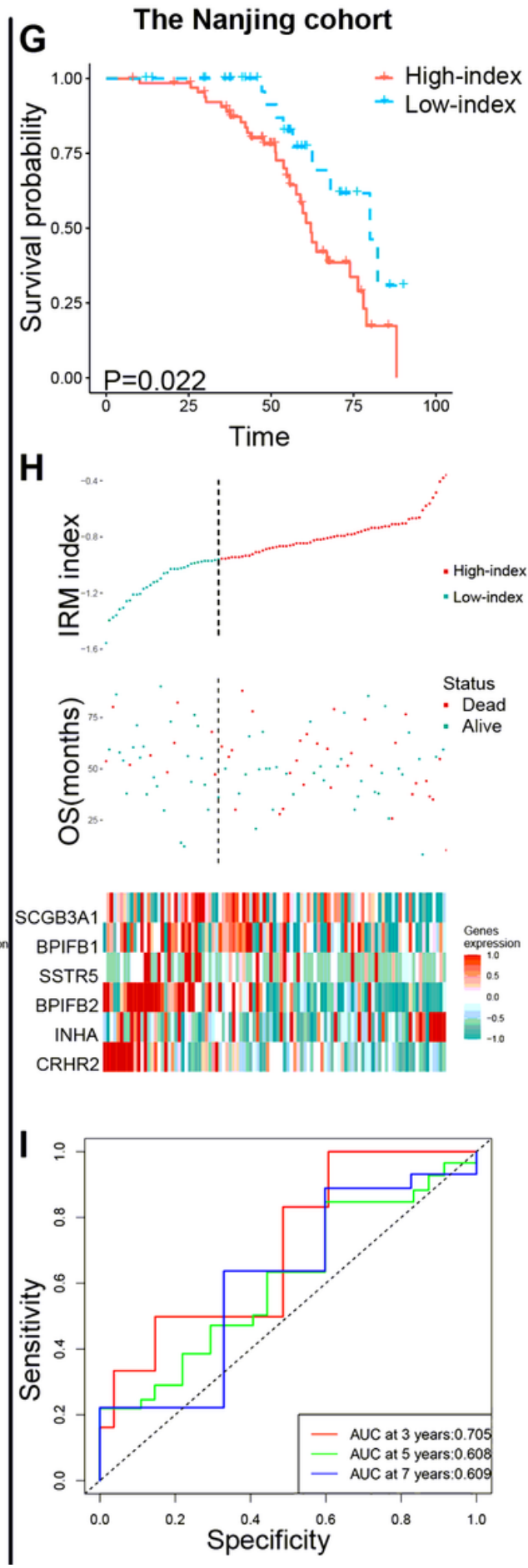

\section{Figure 3}

Prognostic analysis of the Immune-Related Models Index (IRM index). Kaplan-Meier survival, risk score and time-dependent ROC curves of TCGA cohort (A-C), meta-GEO cohort (D-F) and the Jiangsu Cancer Hospital cohort (G-I). (A, D and G) OS was significantly higher in the low-index group than in high-index group. (B, E and H) Relationship between the risk score(upper), the OS (middle) and the expression of six prognostic immune genes(bottom) is shown. (C, F and I) Time-dependent ROC curve analysis of the IRM index. 

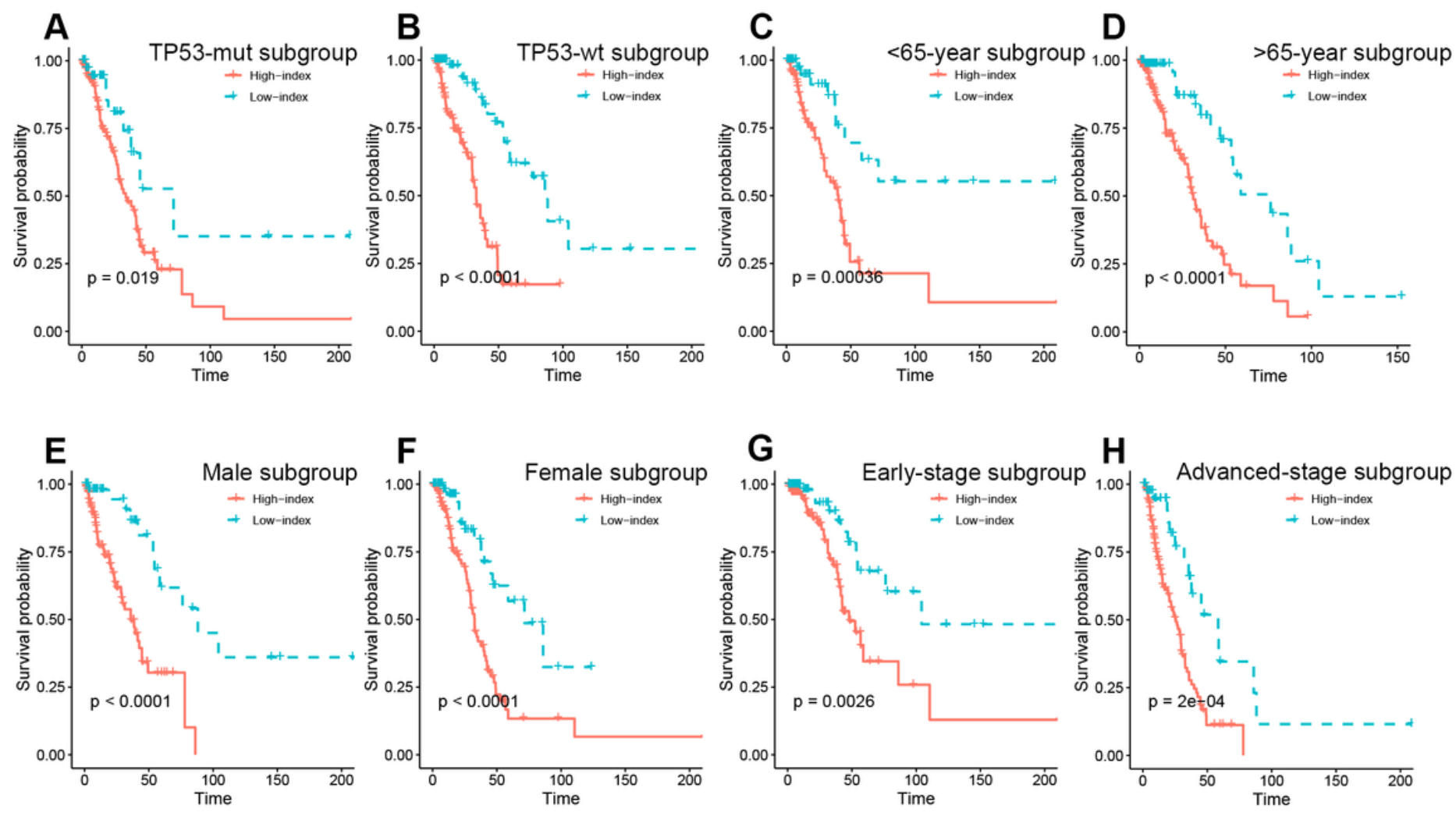

\section{Figure 4}

Stratification analysis. The Kaplan-Meier analysis of the IRM grouping according to patients with (A) TP53 mutant, (B) TP-53 wildtype, (C) <65 years, (D) >65 years, (E) male, (F) female, (G) early stage (TNM stage I), (H) advanced stage (TNM stage II, III, IV). 

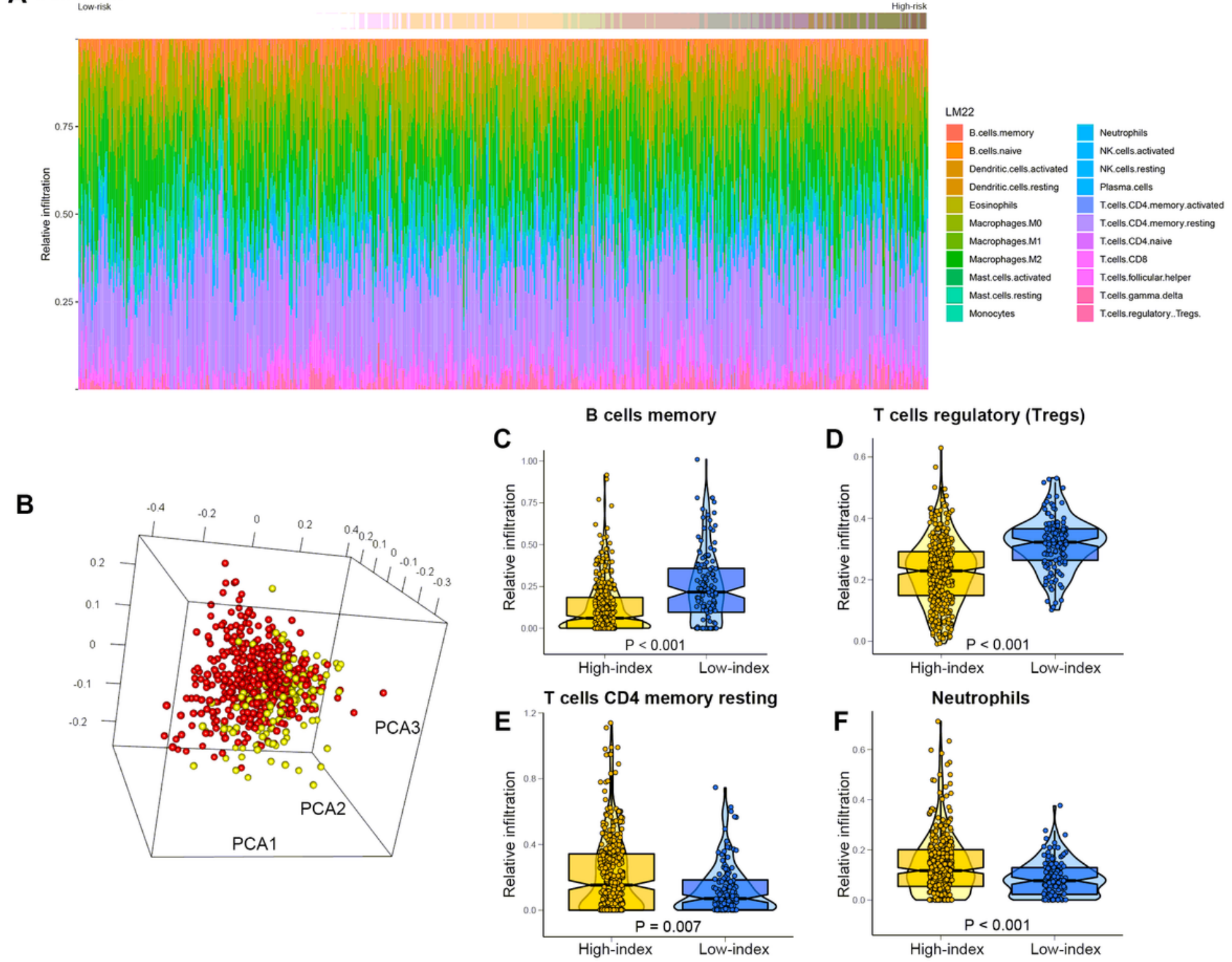

\section{Figure 5}

The landscape of immune infiltration in high- and low-index LUAD patients. (A)Relative infiltration of all 22 immune cells in high- and low-index patients. (B) Principal components analysis performed on LUAD patients based on significant differences in immune cells between high-index and low-index LUAD patients. Box-Violin plots visualizing significantly different immune cells: (C) Memory B cells, (D) Regulatory T cells (Tregs), (E) Resting memory CD4+ T cells, (F) Neutrophils cells. The test for association between paired samples used Pearson's correlation coefficient. Two-tailed statistical $P$ values were calculated by a two-sample Mann-Whitney test or Student's t test when appropriate. 
A

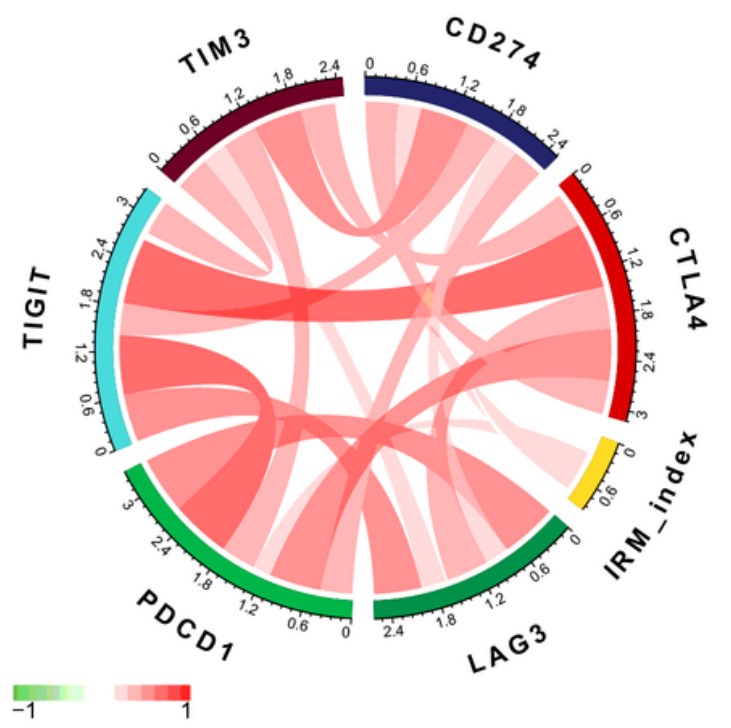

$\mathbf{F}$
B

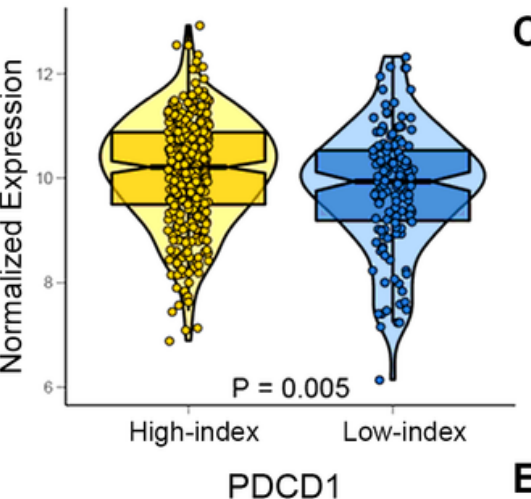

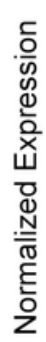

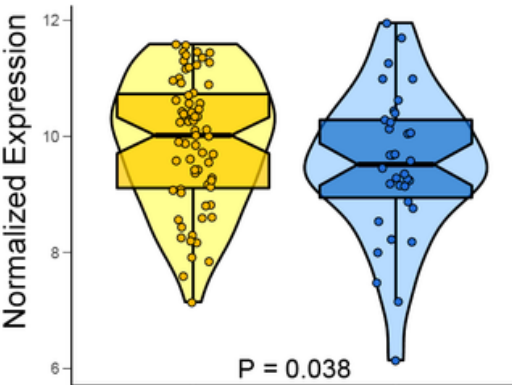

High-index Low-index

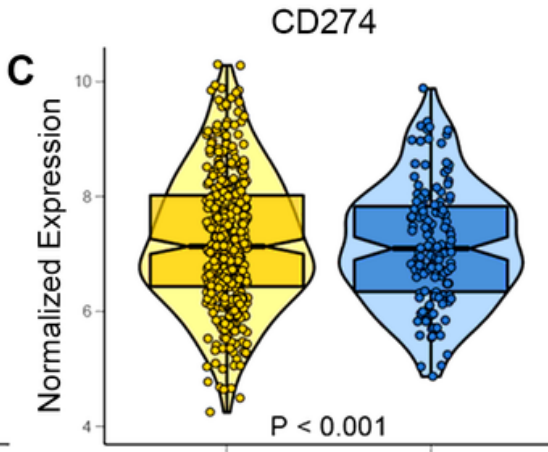

High-index Low-index

E

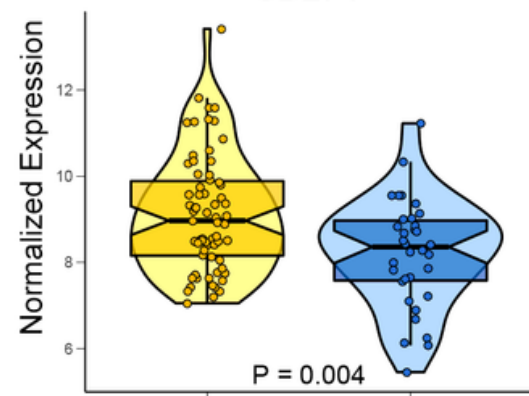

High-index Low-index

PD-1 protein IHC

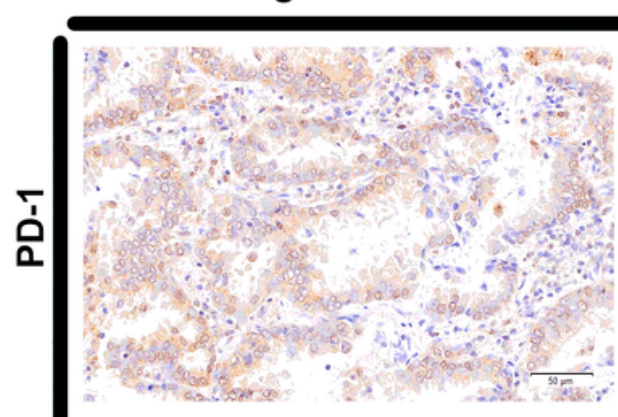

Low-index

G
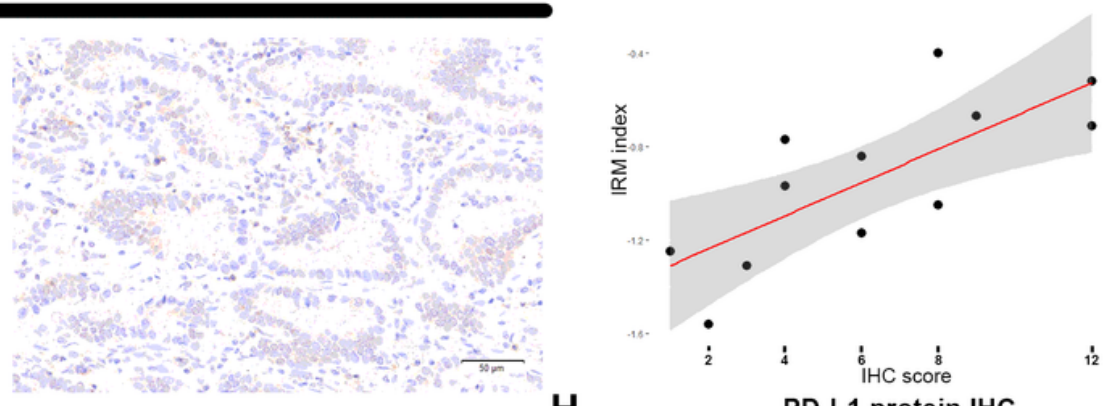

H

PD-L1 protein IHC

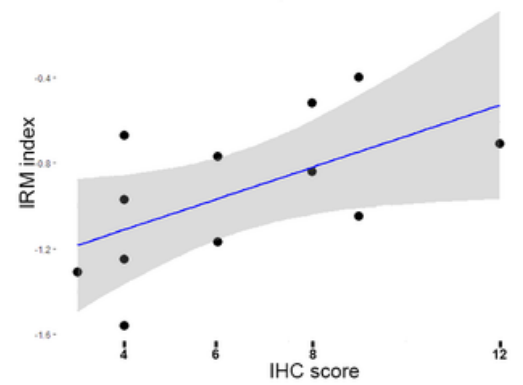

Figure 6

The different expression of immune-checkpoints between high- and low- index LUAD patients. (A) Correlation of the IRM index with the expression of several prominent immune-related checkpoints in TCGA cohort patients. Box-Violin plots visualizing significantly differently expressed immune-related checkpoints: (B) PDCD1 expressed and (C) CD274 expressed. Expression of (D) PDCD1 and (E) CD274 between high- and low-index patients in clinical cohort. (F) Representative images of IHC staining of PD-1 
and PD-L1 in 18 LUAD samples from the Jiangsu Cancer Hospital cohort. (G) $(H)$ The correlation between IRM index and IHC score of the PD-1, PD-L1 protein expression.

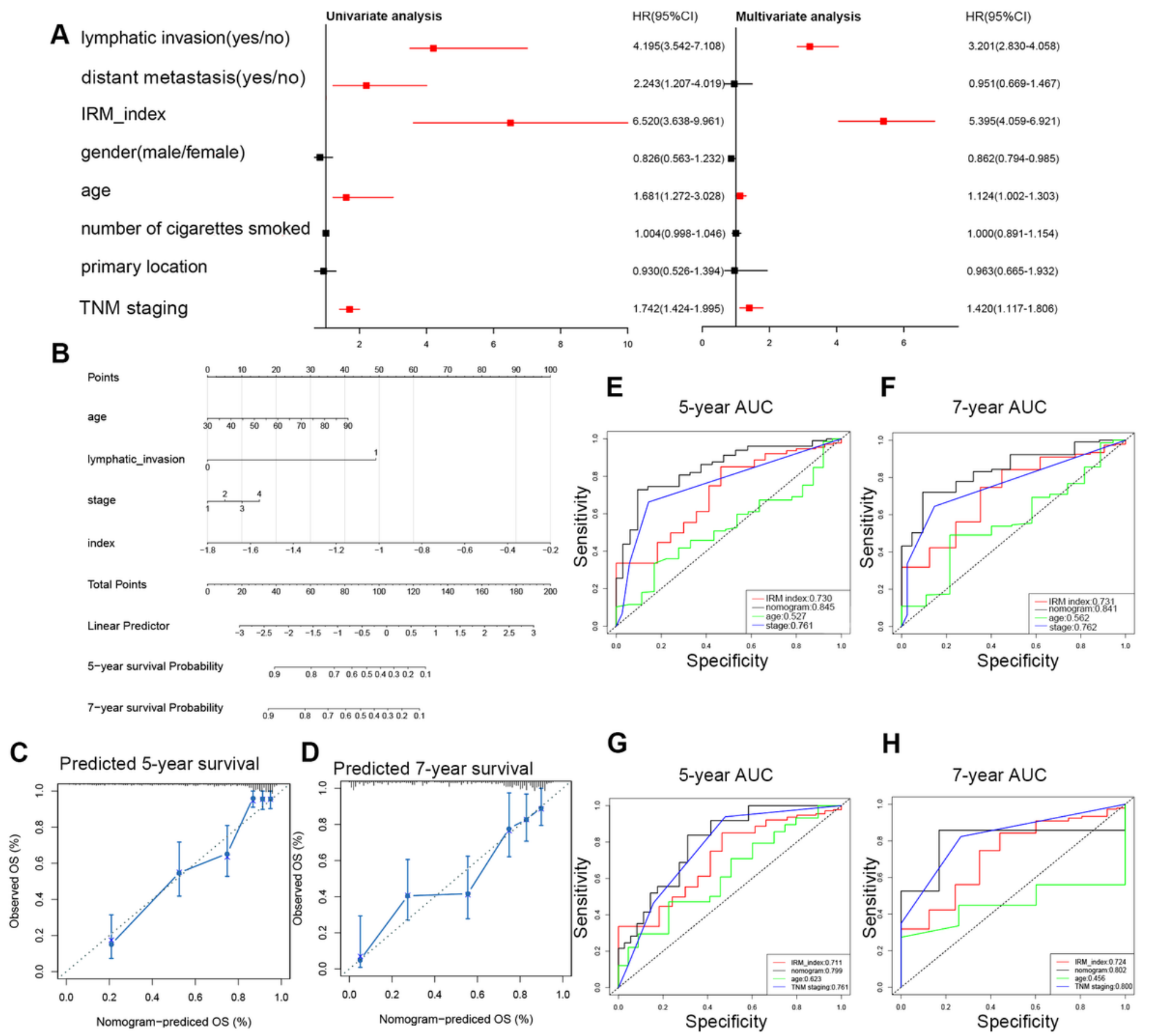

\section{Figure 7}

Construction and validation of the nomogram model. (A) Univariate cox regression analyses and (B) multivariate cox regression analyses for lung adenocarcinoma patients in the TCGA cohort. Red indicates statistical significance (P-value< 0.05), and black indicates no statistical significance. (B) Nomogram for predicting the probability of 5- and 7-year OS for lung adenocarcinoma patients of TCGA cohort. The 
calibration curve of the nomogram for predicting of OS at 5- (C) and 7-year (D). Time-dependent ROC curve analyses of 4 factors, including age, TNM stage, IRM index and nomogram, in 5 years (E) and 7 years $(F)$ in the TCGA cohort. Validation of time-dependent ROC curve analyses of 4 factors in 5 years $(G)$ and 7 years $(G)$ in the Jiangsu Cancer Hospital cohort.

\section{Supplementary Files}

This is a list of supplementary files associated with this preprint. Click to download.

- Tables1.docx

- TableS2.xIsx

- TableS3.xIsx

- TableS4.xIsx

- TableS5.xlsx

- figures1.tif

- figures2.tif

- figures3.tif 